\title{
Improvement of LV functional performance in the chronic total coronary occlusion during the late stage is associated with the extensive collateral development
}

\author{
Yuesong Yang ${ }^{1 *}$, Bradley Strauss ${ }^{1}$, Beiping Qiang ${ }^{1}$, Azriel Osherov¹, John J Graham², Garry Liu', Xiuling Qi ${ }^{1}$, \\ Nigel R Munce', Michelle Ladouceur-Wodzk', Normand Robert ${ }^{1}$, Alexander J Dick', Graham A Wright ${ }^{1}$
}

From 2011 SCMR/Euro CMR Joint Scientific Sessions

Nice, France. 3-6 February 2011

\section{Purpose}

To investigate whether left ventricular (LV) function and regional wall motion improvement are associated with the extent of collateral development in coronary chronic total occlusion (CTO).

\section{Methods}

In nine pigs a $\mathrm{CTO}$ was created by percutaneously inserting a fibrin plug (AngiosealTM) into the mid-todistal left anterior descending artery (LAD). Animals were studied six $(n=3)$ or twelve weeks $(n=6)$ later prior to sacrifice. An x-ray angiogram confirmed the LAD CTO at those time points. Cardiac MR (CMR) studies were then conducted on a $1.5 \mathrm{~T}(\mathrm{n}=6)$ or on a 3.0T MRI system $(\mathrm{n}=3)$, which included SSFP short axis sections for wall motion and post-gadolinium LGE-MRIs for viability. After sacrifice, both right and left coronary systems were injected with Microfil. X-ray or MSCT angiography of the fixed heart was obtained. A longitudinal cardiac section including the CTO lesion, proxi$\mathrm{mal} /$ distal LAD and the borders of infarction was removed and prepared in gel, then imaged in a micro$\mathrm{CT}$ system at 27 micron resolution. LV functional parameters including wall thickness at end-systole (WTES) and end-diastole (WTED) were measured in border zone, infarct and remote region. Systolic wall thickening (SWT) was calculated using as (WTES-WTED) x100/ WTED. CMR and micro-CT data were processed using commercial software. The extent of collaterals on
micro-CT images was rated qualitatively using a score from 0 to 3 , indicating that no, minimal, moderate, or extensive collaterals were observed. A Student's t-test was used for the statistical significance of differences between measurements at 6 and 12 weeks.

\section{Results}

LGE-MRI determined the presence of LV myocardial infarction (MI). Tables 1 and 2 summarize the results of global and regional LV function measurements at both time points. Ejection fraction (LVEF) at 12 weeks was significantly greater than at 6 weeks $(39.45 \pm 5.38 \%$ vs. $26.27 \pm 5.77 \%, \mathrm{P}=0.01$ ) although the extent of infarct was similar between these two groups $(\mathrm{P}=0.16)$. In border zone the WTES $(11.31 \pm 1.72$ vs. $8.67 \pm 0.57 \mathrm{~mm}, \mathrm{P}=0.04)$ and SWT $(68.31 \pm 11.55 \%$ vs. $35.87 \pm 19.14 \%, P=0.01)$ increased at 12 versus 6 weeks. On the visual scores of collateral development, between 6 and 12 weeks, there was an increase in collateral number $(1.33 \pm 0.58$ vs. 2.83 $\pm 0.41, \mathrm{p}<0.003$ ). Figure 1 similarly illustrates increased collateral development at the later time point.

\section{Conclusions}

Extensive collateral development during the late stage of myocardial repair after CTO may contribute to LV functional improvement through increased SWT in the border zone. This provides a potential explanation for preserved LV function witnessed in some patients with CTO. 
Table $1 \mathrm{KV}$ function measurements in coronary CTO pigs

\begin{tabular}{|c|c|c|c|c|c|c|c|c|}
\hline CTO duration & LVEF (\%) & EDV (ml) & ESV (ml) & SV (ml) & $\mathrm{CO}(\mathrm{L})$ & LVM (g) & LV-MI (g) & $\%$ (M/LVM) \\
\hline 6 weeks $(n=3$, mean $\pm S D)$ & $26.27 \pm 5.77$ & $89.64 \pm 20.59$ & $65.73 \pm 14 / 47$ & $23.90 \pm 8.33$ & $1.61 \pm 0.67$ & $71.63 \pm 15.39$ & $7.77 \pm 2.75$ & $11.45 \pm 5.67$ \\
\hline 12 weeks $(n=6$, mean \pm SD) & $39.45 \pm 5.38$ & $185.90 \pm 13.16$ & $113.38 \pm 14.51$ & $73.60 \pm 9.60$ & $5.71 \pm 1.21$ & $129.11 \pm 10.06$ & $10.56 \pm 2.44$ & $8.21 \pm 1.96$ \\
\hline$P$ value & 0.0115 & 0.0001 & 0.0324 & 0.0001 & 0.0011 & 0.0002 & 0.1637 & 0.2351 \\
\hline
\end{tabular}

Note: CTO-Chronic total occlusion, LVEF-Left ventricular ejection fraction, EDV-End-diastolic volume, ESV-End-systolic volume, SV-Stroke volume, CO-Cardiac output, LVM-Left ventricular mass at end-diastolic phase, LV-MI-Myocardial infarction mass.

Table 2 Regional LV wall thickness and systolic wall thickening in coronary CTO pigs

\begin{tabular}{|c|c|c|c|c|c|c|c|c|}
\hline СТO duration & $\begin{array}{l}\text { B-WTES } \\
(\mathrm{mm})\end{array}$ & $\begin{array}{l}\text { B-WTED } \\
(\mathrm{mm})\end{array}$ & $\begin{array}{l}\text { B-Wall } \\
\text { thickening }\end{array}$ & $\begin{array}{l}\text { I-WTES } \\
(\mathrm{mm})\end{array}$ & $\begin{array}{l}\text { I-WTED } \\
(\mathrm{mm})\end{array}$ & $\begin{array}{l}\text { R-WTES } \\
(\mathrm{mm})\end{array}$ & $\begin{array}{l}\text { R-WTED } \\
(\mathrm{mm})\end{array}$ & $\begin{array}{l}\text { R-Wall thickening } \\
\text { (\%) }\end{array}$ \\
\hline $\begin{array}{l}6 \text { weeks }(n=3 \text {, mean } \\
\pm S D)\end{array}$ & $8.67 \pm 0.57$ & $6.51 \pm 1.36$ & $35.87 \pm 19.14$ & $3.19 \pm 0.49$ & $2.82 \pm 0.45$ & $13.54 \pm 2.16$ & $8.38 \pm 1.55$ & $62.1 \pm 5.10$ \\
\hline $\begin{array}{l}12 \text { weeks }(n=6 \text {, mean } \\
\pm S D)\end{array}$ & $11.31 \pm 1.72$ & $6.78 \pm 1.31$ & $68.31 \pm 11.55$ & $2.43 \pm 0.64$ & $2.07 \pm 1.02$ & $13.89 \pm 3.22$ & $8.72 \pm 1.74$ & $58.69 \pm 9.58$ \\
\hline$P$ value & 0.0403 & 0.7834 & 0.0142 & 0.1186 & 0.2756 & 0.8710 & 0.7828 & 0.5881 \\
\hline
\end{tabular}

Note: CTO-Chronic total occlusion, B-Border zone, I-Infarction segment, R-Remote region, WTES-End-systolic wall thickness, WTED-End-diastolic wall thickness.

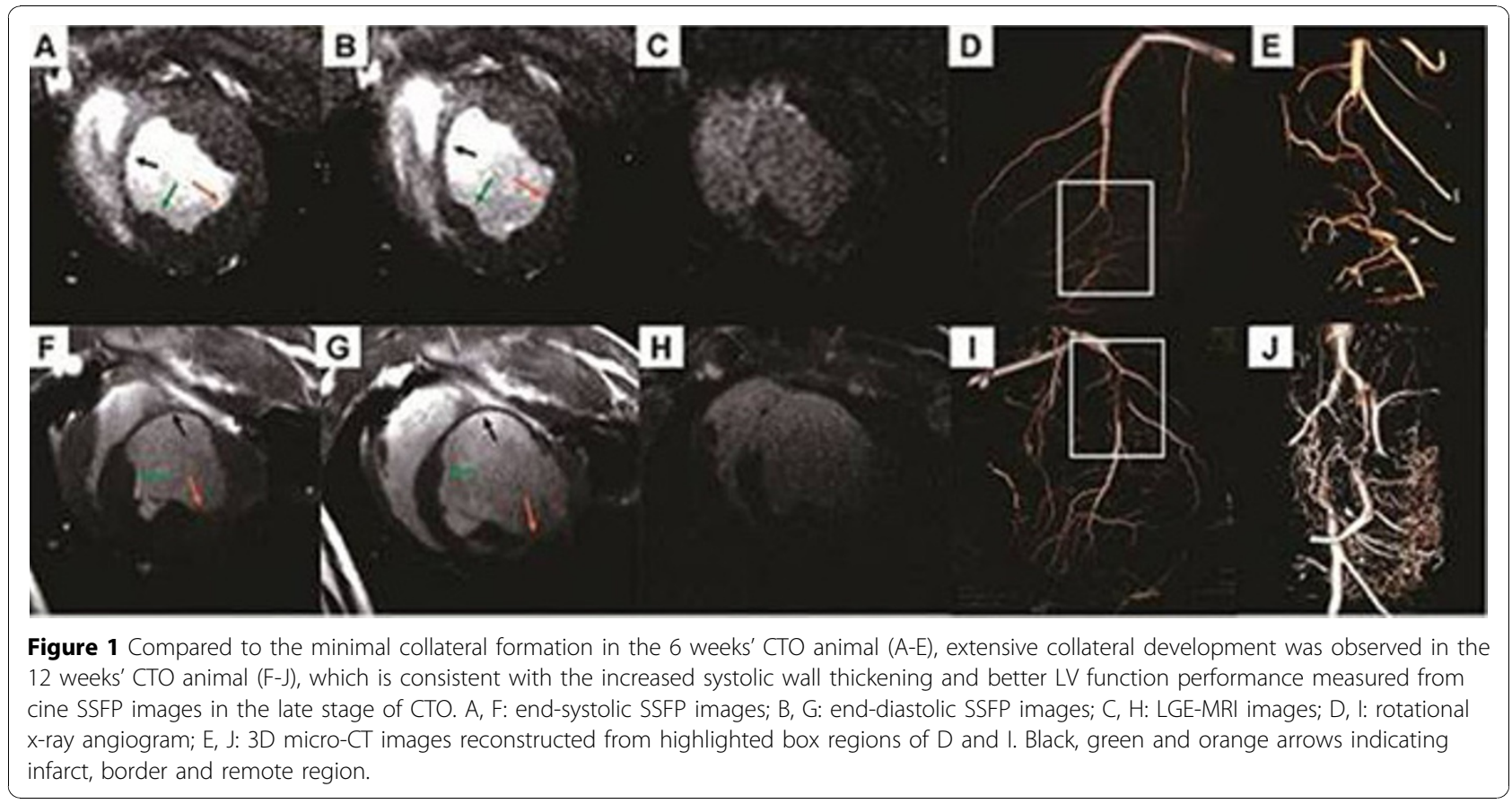

Author details

${ }^{1}$ Sunnybrook Health Sciences Centre, Toronto, ON, Canada. ${ }^{2}$ St.Michael Hospital, Toronto, ON, Canada. ${ }^{3}$ Ottawa Heart Institute, Toronto, ON, Canada.

Published: 2 February 2011

doi:10.1186/1532-429X-13-S1-052

Cite this article as: Yang et al:: Improvement of LV functional performance in the chronic total coronary occlusion during the late stage is associated with the extensive collateral development. Journal of Cardiovascular Magnetic Resonance 2011 13(Suppl 1):O52.
Submit your next manuscript to BioMed Central and take full advantage of:

- Convenient online submission

- Thorough peer review

- No space constraints or color figure charges

- Immediate publication on acceptance

- Inclusion in PubMed, CAS, Scopus and Google Scholar

- Research which is freely available for redistribution 